\title{
Erratum
}

\section{One-Pot Synthesis of 3,4-Dihydro-2H-pyrido[1,2-a][1,3,5]triazin-2-one Derivatives from $N$-(2'-Pyridinyl)benzoylacetamide and Nitrosobenzenes}

Barbara Zaleska,* Bartosz Trzewik, Ewa Stodolak, Jacek Grochowski, Paweł Serda Synthesis 2004, 2975.

Authors' names in the following references were misprinted:

(1) (b) Pawlak, D.; Adamkiewicz, M.; Małyszko, J.; Takada, A.; Myśliwiec, M.; Buczko W. J. Cardiovasc. Pharmacol. 1998, 32, 266.

(1) (c) Pawlak, D.; Pawlak, K.; Chabielska, E.; Małyszko, J.; Takada, A.; Myśiwiec, M.; Buczko, W. Thromb. Res. 1998, 90, 259.

(3) (a) Stanovnik, B.; Tišler, M. Synthesis 1972, 308.

(3) (d) Marchalín, M.; Světlík, J.; Martvoň, A. Collect. Czech. Chem. Commun. 1981, 46, 2557.

The editorial office apologizes for these printing errors. 